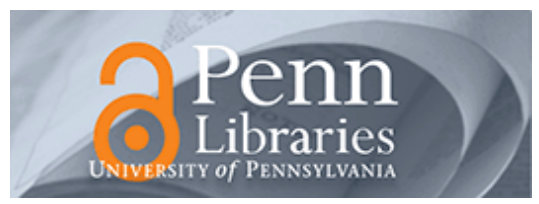

University of Pennsylvania

ScholarlyCommons

January 2008

\title{
An analytic model for the dynamics of electron trapping materials with applications in nonlinear optical signal processing
}

\author{
Ramin Pashaie \\ University of Pennsylvania, raminp@seas.upenn.edu \\ Nabil H. Farhat \\ University of Pennsylvania, farhat@seas.upenn.edu
}

Follow this and additional works at: https://repository.upenn.edu/ese_papers

\section{Recommended Citation}

Ramin Pashaie and Nabil H. Farhat, "An analytic model for the dynamics of electron trapping materials with applications in nonlinear optical signal processing", . January 2008.

This paper is to be published in Journal of the Optical Society of America B, Volume 25, Issue 1: January 2008, pages 15-21, and is made available as an electronic reprint with the permission of OSA. The paper can be found at

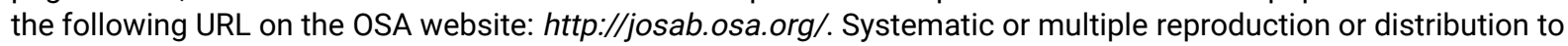
multiple locations via electronic or other means is prohibited and is subject to penalties under law.

This paper is posted at ScholarlyCommons. https://repository.upenn.edu/ese_papers/311

For more information, please contact repository@pobox.upenn.edu. 


\title{
An analytic model for the dynamics of electron trapping materials with applications in nonlinear optical signal processing
}

\begin{abstract}
In this paper the optical mechanism and dynamics of electron trapping material under simultaneous illumination with two wavelengths is investigated. Our analytical model proves that the equilibrium state luminescence of such a material can be controlled to produce highly nonlinear behavior with potential applications in nonlinear optical signal processing and optical realization of nonlinear dynamical systems. Combining this new approach with state-of-the-art fast spatial light modulators and CCD cameras that can precisely control and measure exposure, large arrays of nonlinear processing elements can be accommodated in a thin film of this material.

\section{Comments}

This paper is to be published in Journal of the Optical Society of America B, Volume 25, Issue 1: January 2008 , pages 15-21, and is made available as an electronic reprint with the permission of OSA. The paper

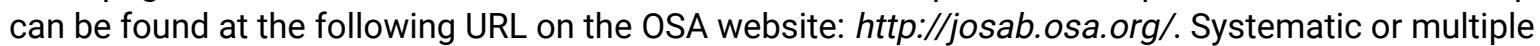
reproduction or distribution to multiple locations via electronic or other means is prohibited and is subject to penalties under law.
\end{abstract}


To be published in Journal of the Optical Society of America B:

Title: An analytic model for the dynamics of electron trapping materials with applications in nonlinear optical signal processing

Authors: Ramin Pashaie and Nabil Farhat

Accepted: 24 October 2007

Posted: 6 November 2007

Doc. ID: $\quad 84630$ 


\title{
An analytic model for the dynamics of electron 1
}

\section{trapping materials with applications in nonlinear}

\section{optical signal processing}

\author{
Ramin Pashaie, ${ }^{1, *}$ and Nabil H. Farhat, ${ }^{1,2}$ \\ ${ }^{1}$ Department of Electrical and System Engineering, University of Pennsylvania, \\ 200 South 33rd Street, Philadelphia, Pennsylvania 19104-6391, USA \\ ${ }^{2}$ Mahoney Institute of Neurological Sciences, University of Pennsylvania, \\ 3450 Hamilton Walk, Philadelphia, PA 19104-60\%4, USA
}

*Corresponding author: raminp@seas.upenn.edu 
In this paper the optical mechanism and dynamics of electron trapping material under simultaneous illumination with two wavelengths is investigated. Our analytical model proves that the equilibrium state luminescence of such a material can be controlled to produce highly nonlinear behavior with potential applications in nonlinear optical signal processing and optical realization of nonlinear dynamical systems. Combining this new approach with state-of-theart fast spatial light modulators and CCD cameras that can precisely control and measure exposure, large arrays of nonlinear processing elements can be accommodated in a thin film of this material.

(c) 2007 Optical Society of America

OCIS codes: 200.0200, 200.3050, 200.4560, 200.4740

\section{Introduction}

Optical signal processing (OSP) compared to other conventional methods of data processing, such as digital signal processing (DSP), has some advantages [1]. For instance, realization of parallelism and massive interconnections can be done far better in optics than electronics and digital circuits that are good in serial processing of segmented information. Nevertheless, in many application of OSP we need nonlinear optical devices whose degree of nonlinearity is more than saturation. For example, consider the simple optical arrangement in Fig. 1(a). A photodetector measures the 
intensity of the light emitted from a light source and passed through an optical device 3 . Usually, the output voltage of the photodetector below saturation is a linear function of the intensity of illumination. However, in many applications (e.g. optical realization of one-dimensional maps such as quadratic return map or logistic map in nonlinear dynamics [2]) we require a nonlinear behavior same as the desired curve depicted in Fig. 1(b). Here, the intensity of the detected light increases at first and decreases when we pass a maximum point. To our knowledge an optical component with such nonlinear response is not available. Recently, it has been shown experimentally that the equilibrium state luminescence of electron trapping materials (ETMs) can be controlled to exhibit this type of nonlinearity [3,4]. In this paper, we analytically study the same problem and derive sufficient conditions that should be satisfied by the ETM parameters so that the material would be capable of producing the desired nonlinear curves in the equilibrium state. Also we describe a systematic way to reduce the time constant of the process so that the ETM reaches the equilibrium state faster.

\section{Physics and dynamics of ETMs}

The energy band diagram of ETM and its optical mechanism is shown in Fig. 2. This system contains an electron-rich valance band, a conduction band and a trap energy level that is almost empty for an ETM in a dark room with no external stimulus. Now, suppose that the material is illuminated by two sources of light with wavelengths $\lambda_{1}$ and $\lambda_{3}$ where $\lambda_{1}<\lambda_{3}$. Photons with wavelength $\lambda_{1}$ have enough energy to interact 
with electrons of the valance band and excite them to the conduction band. Some of the excited electrons tunnel to the trap energy level and become trapped electrons. Other excited electrons return to the valance band to release their extra energy in the form of spontaneous emission with wavelength $\lambda_{2}$ such that $\lambda_{1}<\lambda_{2}<\lambda_{3}$. $\lambda_{1}$ photons can also interact with trapped electrons to kick them out of the traps to the conduction band. These electrons can return to the valance band and produce luminescence or they can tunnel to the trap levels. If these electrons return to the trap levels there would be no change in the population of the trapped electrons and also no luminescence. Illuminating the material with wavelength $\lambda_{3}$ detraps some of the trapped electrons and generates luminescence. The band gap between the trap energy level and the conduction band should be small enough so that interaction of trapped electrons and photons of wavelength $\lambda_{3}$ can excite the trapped electron to the conduction band and wide enough so that electron-phonon interactions would not be able to detrap the trapped electrons [5]. An analogue dynamics in fluid mechanics is depicted in the system of water tanks displayed in Fig. 3.

A well-known ETM is $S r S: E u^{2+} S m^{3+}$ which is an alkaline-earth sulfides doped with rare-earth luminescence centers. Rare-earth doped elements add a trap energy level within the host bandgap. Exposing such ETM to blue light of wavelength $\lambda_{1}=450 \mathrm{~nm}$ increases the density of trapped electrons and near infrared exposure of wavelength $\lambda_{3}=1310 \mathrm{~nm}$ detraps trapped electrons. Luminescence of this material is orange light with peak wavelength around $\lambda_{2}=650 \mathrm{~nm}$. This infrared stimulable 
storage phosphor has been widely studied for applications in optical computing [6-8 $\bar{p}$,

optical data storages [9], and biology inspired models $[4,10]$.

\section{Equations}

Before formulating the mathematical model let us review the nomenclature that is used in this paper.
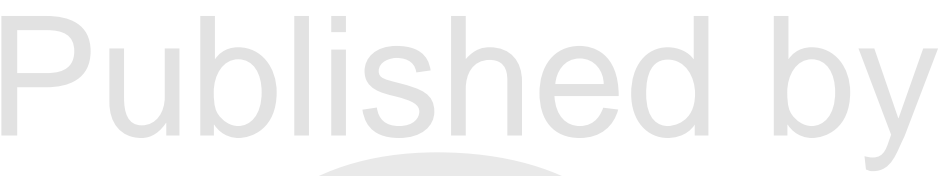
$\xi_{v \rightarrow c}^{1} \quad$ Probability of transition from the valance band to the conduction band caused by interaction of electrons in the valance band and $\lambda_{1}$ photons,

$\xi_{t \rightarrow c}^{1} \quad$ Probability of transition from the trap energy level to the conduction band (detrapping) caused by interaction of the trapped electrons and $\lambda_{1}$ photons, $\xi_{t \rightarrow c}^{3} \quad$ Probability of transition from the trap energy level to the conduction band (detrapping) caused by interaction of the trapped electrons and $\lambda_{3}$ photons, $\xi_{c \rightarrow t}^{T}$ Probability of tunneling from the conduction band to the trap energy level, $\xi_{c \rightarrow v}^{R}$ Probability of relaxation from the conduction band to the valance band, $\xi_{\text {pass }}^{1} \quad$ Probability of passing through the material without absorption for a photon with wavelength $\lambda_{1}$,

$\xi_{\text {pass }}^{3} \quad$ Probability of passing through the material without absorption for a photon with wavelength $\lambda_{3}$,

$I_{i} \quad$ Intensity of light with wavelength $\lambda_{i}, i=1,2,3,(\mathrm{~W})$

$P_{i}^{p h} \quad$ Number of incident photons with wavelength $\lambda_{i}, i=1,2,3,(1 / \mathrm{s})$ (flow of $\lambda_{i}$ photons),

$P_{v}^{e} \quad$ Population of electrons in the valance band,

$P_{t}^{e} \quad$ Population of electrons in the trap level,

$P^{e} \quad$ Total population of electrons in the valance band and trap energy level $P^{e}=P_{t}^{e}(t)+P_{v}^{e}(t)$. In writing this expression we assumed that the lifetime for an electron in the conduction band is so short that once an electron is excited up to the conduction band, it almost immediately relaxes to the valance band or tunnels to the trap energy level. Regarding the millisecond time-scale that we use 
There are few relations between the probability variables that we defined above? When we expose the ETM to the light with wavelength $\lambda_{1}$, these photons interact with the electrons or they pass through the ETM thin film without absorbtion which means that: $\xi_{v \rightarrow c}^{1}+\xi_{t \rightarrow c}^{1}+\xi_{p a s s}^{1}=1$. Similarly, for the photons with wavelength $\lambda_{3}: \xi_{t \rightarrow c}^{3}+$ $\xi_{\text {pass }}^{3}=1$. For an incident photon, the probability of electron-photon interaction in the valance and trap band is proportional to the population of electrons in each energy level: $\xi_{v \rightarrow c}^{1} \propto P_{v}^{e}(t), \xi_{t \rightarrow c}^{1} \propto P_{t}^{e}(t)$, and $\xi_{t \rightarrow c}^{3} \propto P_{t}^{e}(t)$. These proportionalities can be changed to equalities by defining the proportionality coefficients $0 \leq \kappa_{v}^{1}, \kappa_{t}^{1}, \kappa_{t}^{3} \leq 1.0$, so that: $\xi_{v \rightarrow c}^{1}=\kappa_{v}^{1} P_{v}^{e}(t) / P^{e}, \xi_{t \rightarrow c}^{1}=\kappa_{t}^{1} P_{t}^{e}(t) / P^{e}$, and $\xi_{t \rightarrow c}^{3}=\kappa_{t}^{3} P_{t}^{e}(t) / P^{e}$. Once an electron is excited up to the conduction band this electron can relax to valance band or it can tunnel to the trap energy level which means that: $\xi_{c \rightarrow t}^{T}+\xi_{c \rightarrow v}^{R}=1$.

Consider the case where an ETM is under constant and continuous simultaneous illumination with wavelengths $\lambda_{1}$ and $\lambda_{3}$. Our goal is to compute the intensity of luminescence as a function of the intensities of the two illuminations. We start by writing the equations for the populations of electrons in the valance band and trap level as a function of time, $P_{v}^{e}(t)$ and $P_{t}^{e}(t)$, and same populations a tiny amount of time before, $P_{v}^{e}(t-\delta)$ and $P_{t}^{e}(t-\delta)$.

$$
\begin{aligned}
P_{v}^{e}(t)=P_{v}^{e}(t-\delta)- & \delta P_{1}^{p h} \xi_{v \rightarrow c}^{1} \xi_{c \rightarrow t}^{T} \\
& +\delta P_{1}^{p h} \xi_{t \rightarrow c}^{1} \xi_{c \rightarrow v}^{R}+\delta P_{3}^{p h} \xi_{t \rightarrow c}^{3} \xi_{c \rightarrow v}^{R}, \\
P_{t}^{e}(t)=P_{t}^{e}(t-\delta)+ & \delta P_{1}^{p h} \xi_{v \rightarrow c}^{1} \xi_{c \rightarrow t}^{T}
\end{aligned}
$$




$$
-\delta P_{1}^{p h} \xi_{t \rightarrow c}^{1} \xi_{c \rightarrow v}^{R}-\delta P_{3}^{p h} \xi_{t \rightarrow c}^{3} \xi_{c \rightarrow v}^{R} .
$$

The intensity of light with wavelength $\lambda_{i}$ can be computed from the number of incident photons per unit of time, $P_{i}^{p h}$, using the equation: $I_{i}=P_{i}^{p h} h \nu_{i}$ where $h$ is the Planck constant and $\nu_{i}$ is the frequency of radiation. If $\delta \rightarrow 0$ and we divide both side of the equations by $P^{e}$, we get the normalized rate of changes in the electron populations.

$$
\begin{aligned}
& \frac{d P_{v}^{e}(t)}{d t}=-\frac{\kappa_{v}^{1} \xi_{c-t}^{T}}{P^{e}} P_{1}^{p h} P_{v}^{e}(t)+\frac{\kappa_{t}^{1} \xi_{c-v}^{R}}{P^{e}} P_{1}^{p h} P_{t}^{e}(t)+\frac{\kappa_{t}^{3} \xi_{c \rightarrow v}^{R}}{P^{e}} P_{3}^{p h} P_{t}^{e}(t), \\
& \frac{d P_{t}^{e}(t)}{d t}=+\frac{\kappa_{v}^{1} \xi_{c \rightarrow t}^{T}}{P^{e}} P_{1}^{p h} P_{v}^{e}(t)-\frac{\kappa_{t}^{1} \xi_{c-v}^{R}}{P^{e}} P_{1}^{p h} P_{t}^{e}(t)-\frac{\kappa_{t}^{3} \xi_{c-v}^{R}}{P^{e}} P_{3}^{p h} P_{t}^{e}(t) .
\end{aligned}
$$

and as we expect: $d P_{t}^{e} / d t=-d P_{v}^{e} / d t$. The number of luminescent photons, $P_{2}^{p h}(t)$, is:

$$
P_{2}^{p h}(t)=\frac{\kappa_{v}^{1} \xi_{c-v}^{R}}{P^{e}} P_{1}^{p h} P_{v}^{e}(t)+\frac{\kappa_{t}^{1} \xi_{c \rightarrow v}^{R}}{P^{e}} P_{1}^{p h} P_{t}^{e}(t)+\frac{\kappa_{t}^{3} \xi_{c-v}^{R}}{P^{e}} P_{3}^{p h} P_{t}^{e}(t)
$$

Typical charging characteristic curves where $I_{1}>0$ and $I_{3}=0$, and discharging characteristic curves where $I_{1}=0$ and $I_{3}>0$ for the described electron trapping material are given in Fig. 4. As we can see in Fig 4(a), charging curves rise up rapidly and converge to a saturation level that depends on $I_{1}$. This saturation level exists because during charging process two opposing processes of trapping and detrapping are taking place simultaneously and the saturation level is the balance point. On the other hand, during discharging the only ongoing process is detrapping and all the curves converge to zero (Fig. 4(b)). Similar to the experimental results previously 
reported [3], the level of the initial jumps in both charging and discharging curves and the final saturation level in the charging curves are linear functions of the intensities of exposure in each case.

A more interesting situation in the optical mechanism of electron trapping materials occurs when the material remains under constant simultaneous illumination and the intensity of luminescence converges to its equilibrium state emission. At equilibrium $d P_{t}^{e}(t) / d t=-d P_{v}^{e}(t) / d t=0$ so the equilibrium condition as deduced from equations (3) and (4) will be,

$$
\kappa_{v}^{1} \xi_{c \rightarrow t}^{T} P_{1}^{p h} \tilde{P}_{v}^{e}=\kappa_{t}^{1} \xi_{c \rightarrow v}^{R} P_{1}^{p h} \tilde{P}_{t}^{e}+\kappa_{t}^{3} \xi_{c \rightarrow v}^{R} P_{3}^{p h} \tilde{P}_{t}^{e},
$$

where $\tilde{P}_{v}^{e}$, and $\tilde{P}_{t}^{e}$ are respectively the density of electrons in the valance band and trap energy level at the equilibrium state. This equation help us to calculate the way the total population of electrons, $P^{e}$, is split (in the equilibrium state) between the valance band and the trap energy level.

$$
\begin{aligned}
& \tilde{P}_{v}^{e}=\left(\alpha+\frac{P_{3}^{p h}}{P_{1}^{p h}} \beta\right) \tilde{P}_{t}^{e}, \\
& \tilde{P}_{v}^{e}+\tilde{P}_{t}^{e}=P^{e} .
\end{aligned}
$$

In equation (7), $\alpha=\frac{\kappa_{\kappa^{1}}^{1} \xi_{c \rightarrow v}^{R}}{\kappa_{v}^{1} \xi_{c \rightarrow t}^{T}}$, and $\beta=\frac{\kappa_{t}^{3} \xi_{c t v}^{R}}{\kappa_{v}^{1} \xi_{c \rightarrow t}^{T}}$ and $\alpha, \beta>0$. Solving these two equations for $\tilde{P}_{v}^{e}$ and $\tilde{P}_{t}^{e}$ gives,

$$
\begin{aligned}
\tilde{P}_{v}^{e} & =\frac{\alpha P_{1}^{p h}+\beta P_{3}^{p h}}{(1+\alpha) P_{1}^{p h}+\beta P_{3}^{p h}} P^{e}, \\
\tilde{P}_{t}^{e} & =\frac{P_{1}^{p h}}{(1+\alpha) P_{1}^{p h}+\beta P_{3}^{p h}} P^{e} .
\end{aligned}
$$


Using equation (5), the intensity of luminescence in the equilibrium state can not be expressed as follows:

$$
\frac{1}{\kappa_{v}^{1}} \tilde{P}_{2}^{p h}=P_{1}^{p h} \frac{\alpha P_{1}^{p h}+\beta P_{3}^{p h}}{(1+\alpha) P_{1}^{p h}+\beta P_{3}^{p h}}=f\left(P_{1}^{p h}, P_{3}^{p h}\right) .
$$

Equation (11) shows how the intensity of luminescence depends on the intensities of illuminations. In order to investigate this functionality further, let us examine the gradient and the Hessian of the function $f\left(P_{1}^{p h}, P_{3}^{p h}\right)$.

$$
\begin{aligned}
& \nabla f\left(P_{1}^{p h}, P_{3}^{p h}\right) \triangleq \frac{\partial f}{\partial P_{1}^{p h}} \hat{P}_{1}^{p h}+\frac{\partial f}{\partial P_{3}^{p h}} \hat{P}_{3}^{p h} \\
& =\frac{\alpha(1+\alpha)\left(P_{1}^{p h}\right)^{2}+2 \alpha \beta P_{1}^{p h} P_{3}^{p h}+\beta^{2}\left(P_{3}^{p h}\right)^{2}}{\left[(1+\alpha) P_{1}^{p h}+\beta P_{3}^{p h}\right]^{2}} \hat{P}_{1}^{p h}+\frac{\beta\left(P_{1}^{p h}\right)^{2}}{\left[(1+\alpha) P_{1}^{p h}+\beta P_{3}^{p h}\right]^{2}} \hat{P}_{3}^{p h}, \\
& H(f) \triangleq\left[\begin{array}{cc}
\frac{\partial^{2} f}{\partial^{2} P_{1}^{p h}} & \frac{\partial^{2} f}{\partial P_{1}^{p h} \partial P_{3}^{p h}} \\
\frac{\partial^{2} f}{\partial P_{3}^{p h} \partial P_{1}^{p h}} & \frac{\partial^{2} f}{\partial^{2} P_{3}^{p h}}
\end{array}\right]=\frac{2 \beta^{2}}{\left[(1+\alpha) P_{1}^{p h}+\beta P_{3}^{p h}\right]^{3}}\left[\begin{array}{cc}
-\left(P_{3}^{p h}\right)^{2} & P_{1}^{p h} P_{3}^{p h} \\
P_{1}^{p h} P_{3}^{p h} & -\left(P_{1}^{p h}\right)^{2}
\end{array}\right]
\end{aligned}
$$

In equation (12), $\hat{P}_{1}^{p h}$ and $\hat{P}_{3}^{p h}$ are the unit vectors. Obviously, $\partial f / \partial P_{1}^{p h}, \partial f / \partial P_{3}^{p h} \geq$ $0, \forall P_{1}^{p h}, P_{3}^{p h}, \alpha, \beta$. Also it can be proved that the Hessian matrix is semi-negative definite. As a result, the $f\left(P_{1}^{p h}, P_{3}^{p h}\right)$ is a monotonically increasing concave function (Fig. 5(a)) and the level sets of this function in the $P_{1}^{p h}-P_{2}^{p h}$ plane are convex contours similar to the example in Fig. 5(b).

Another important factor is the speed of this system, defined as the speed of the system to arrive at equilibrium. In order to compute the speed of the system we compute the time constant for the evolution of the population of the trapped electron, 
$P_{t}^{e}(t)$, by using equation (4). We can rewrite equation (4) as follows:

$$
\begin{aligned}
& \frac{d P_{t}^{e}(t)}{d t}=\theta-\phi P_{t}^{e}(t) \\
& \theta=\frac{1}{P^{e}} \kappa_{v}^{1} \xi_{c \rightarrow t}^{T} P_{1}^{p h} \\
& \phi=\frac{1}{P^{e}}\left(\kappa_{v}^{1} \xi_{c \rightarrow t}^{T} P_{1}^{p h}+\kappa_{t}^{1} \xi_{c \rightarrow v}^{R} P_{1}^{p h}+\kappa_{t}^{3} \xi_{c \rightarrow v}^{R} P_{3}^{p h}\right) .
\end{aligned}
$$

This differential equation can be solved for $P_{t}^{e}(t)$.

$$
P_{t}^{e}(t)=\frac{\theta}{\phi}-\frac{1}{\phi} \exp (-\phi t)
$$

Accordingly, the time constant of the system is $\tau=1 / \phi$. In order to improve the speed of the system we need to minimize $\tau$ by maximizing $\phi$. Obviously, $\phi \propto 1 / P^{e}$ so that a system in which the total population of electrons is less reaches the equilibrium state faster (the intensity of luminescence in the equilibrium state is independent of $\left.P^{e}\right)$. For the case where $P^{e}$ is constant, this optimization problem can be expressed as follows:

$$
\begin{aligned}
& \text { Maximize }\left(\text { Maximize } P^{e} \phi\right), \\
& \xi_{c \rightarrow v}^{R}, \xi_{c \rightarrow t}^{T}, \kappa_{v}^{1}, \kappa_{t}^{1}, \kappa_{t}^{3} \quad I_{1}, I_{3}
\end{aligned}
$$

Subject to :

$$
\begin{aligned}
& P^{e} \phi=\kappa_{v}^{1} \xi_{c \rightarrow t}^{T} P_{1}^{p h}+\kappa_{t}^{1} \xi_{c \rightarrow v}^{R} P_{1}^{p h}+\kappa_{t}^{3} \xi_{c \rightarrow v}^{R} P_{3}^{p h}, \\
& I_{1} \in\left[I_{1}^{M i n}, I_{1}^{M a x}\right], I_{3} \in\left[I_{3}^{M i n}, I_{3}^{M a x}\right], \\
& P_{i}^{p h}=I_{i} / h \nu_{i},
\end{aligned}
$$




$$
\begin{aligned}
& 0<\xi_{c \rightarrow v}^{R}, \xi_{c \rightarrow t}^{T}, \kappa_{v}^{1}, \kappa_{t}^{1}, \kappa_{t}^{3}<1, \\
& \xi_{c \rightarrow v}^{R}+\xi_{c \rightarrow t}^{T}=1 .
\end{aligned}
$$

Results of this nonlinear optimization can be used for synthesis of luminescent materials. In this problem we are maximizing the speed of the system over two different sets of variables. The first set, $\left\{\xi_{c \rightarrow v}^{R}, \xi_{c \rightarrow t}^{T}, \kappa_{v}^{1}, \kappa_{t}^{1}, \kappa_{t}^{3}\right\}$, includes the material parameters in contrast to the second set, $\left\{I_{1}, I_{3}\right\}$, which are variables under control of the user. Maximization over the second set is a linear optimization. Consequently, the optimal solution should occur on one of the corners of the rectangular interval $I_{1} \in\left[I_{1}^{\text {Min }}, I_{1}^{\text {Max }}\right], I_{3} \in\left[I_{3}^{\text {Min }}, I_{3}^{\text {Max }}\right]$ in the $I_{1}-I_{3}$ plane. If we take the gradient of $P^{e} \phi$ with respect to $I_{1}$ and $I_{3}$ we get: $P^{e} \nabla_{I_{1}, I_{3}} \phi=P^{e} \frac{\partial \phi}{\partial I_{1}} \hat{I}_{1}+P^{e} \frac{\partial \phi}{\partial I_{3}} \hat{I}_{3}$, where $P^{e} \frac{\partial \phi}{\partial I_{1}}=\frac{1}{h \nu_{1}}\left(\kappa_{v}^{1} \xi_{c \rightarrow t}^{T}+\kappa_{t}^{1} \xi_{c \rightarrow v}^{R}\right)>0$ and $P^{e} \frac{\partial \phi}{\partial I_{3}}=\frac{1}{h \nu_{3}}\left(\kappa_{t}^{3} \xi_{c \rightarrow v}^{R}\right)>0$. Hence, the optimal solution for the first maximization problem is: $I_{1}=I_{1}^{M a x}$ and $I_{3}=I_{3}^{\text {Max }}$. From this result we can conclude that more intense exposures causes faster convergence to the equilibrium emission. In the second maximization, increasing each of the variables: $\kappa_{v}^{1}, \kappa_{t}^{1}, \kappa_{t}^{3}$ monotonically increases the speed of the system. If we calculate the derivative of the objective with respect to $\xi_{c \rightarrow v}^{R}$ we get: $P^{e} \frac{\partial \phi}{\partial \xi_{c \rightarrow v}^{R}}=\kappa_{v}^{1} P_{1}^{p h}+\kappa_{t}^{3} P_{3}^{p h}-\kappa_{v}^{1} P_{1}^{p h}$. Therefore, when $\kappa_{v}^{1} P_{1}^{p h}+\kappa_{t}^{3} P_{3}^{p h}>\kappa_{v}^{1} P_{1}^{p h}$ increasing $\xi_{c \rightarrow v}^{R}$ (decreasing $\xi_{c \rightarrow t}^{T}$ ) improves the speed of the system and visa versa.

We may also try to maximize the luminescence intensity in the equilibrium state over the material parameters and in a specific dynamic range of the exposures. This 
maximization can be written as a mathematical programming problem as follows: ${ }^{13}$

$$
\begin{aligned}
& \text { Maximize }\left(\text { Maximize } \tilde{P}_{2}^{p h}\right), \\
& \xi_{c \rightarrow v}^{R}, \xi_{c \rightarrow t}^{T}, \kappa_{v}^{1}, \kappa_{t}^{1}, \kappa_{t}^{3} \quad I_{1}, I_{3}
\end{aligned}
$$

Subject to :

$$
\begin{aligned}
& \tilde{P}_{2}^{p h}=\kappa_{v}^{1} P_{1}^{p h} \frac{\kappa_{t}^{1} P_{1}^{p h} \xi_{c \rightarrow v}^{R}+\kappa_{t}^{3} P_{3}^{p h} \xi_{c \rightarrow v}^{R}}{\left(\kappa_{v}^{1} \xi_{c \rightarrow t}^{T}+\kappa_{t}^{1} \xi_{c \rightarrow v}^{R}\right) P_{1}^{p h}+\kappa_{t}^{3} P_{3}^{p h} \xi_{c \rightarrow v}^{R}}, \\
& I_{1} \in\left[I_{1}^{M i n}, I_{1}^{M a x}\right], I_{3} \in\left[I_{3}^{M i n}, I_{3}^{M a x}\right] \\
& P_{i}^{p h}=I_{i} / h \nu_{i}, \\
& 0<\xi_{c \rightarrow v}^{R}, \xi_{c \rightarrow t}^{T}, \kappa_{v}^{1}, \kappa_{t}^{1}, \kappa_{t}^{3}<1, \\
& \xi_{c \rightarrow v}^{R}+\xi_{c \rightarrow t}^{T}=1 .
\end{aligned}
$$

Clearly, this is a nonlinear optimization with several variables. We have already shown (equation (12)) that the objective is monotonically increasing over the dynamic range of the intensity of exposures. In order to solve the second maximization we need to look at the partial derivatives of the objective with respect to the material parameters.

$$
\begin{aligned}
& \partial \tilde{P}_{2}^{p h} / \partial \xi_{c \rightarrow v}^{R}=\kappa_{v}^{1} P_{1}^{p h}\left(\kappa_{t}^{1} P_{1}^{p h}+\kappa_{t}^{3} P_{3}^{p h}\right) / g^{2} \geq 0 \\
& \partial \tilde{P}_{2}^{p h} / \partial \kappa_{v}^{1}=P_{1}^{p h} \xi_{c \rightarrow v}^{R}\left(\kappa_{t}^{1} P_{1}^{p h}+\kappa_{t}^{3} P_{3}^{p h}\right)\left(\kappa_{t}^{1} P_{1}^{p h} \xi_{c \rightarrow t}^{T}+\kappa_{t}^{3} P_{3}^{p h} \xi_{c \rightarrow v}^{R}\right) / g^{2} \geq 0 \\
& \partial \tilde{P}_{2}^{p h} / \partial \kappa_{t}^{1}=\kappa_{v}^{1}\left(P_{1}^{p h}\right)^{2} \xi_{c \rightarrow v}^{R}\left(\kappa_{v}^{1} P_{1}^{p h} \xi_{c \rightarrow v}^{R}+\kappa_{t}^{3} P_{3}^{p h} \xi_{c \rightarrow v}^{R}-\kappa_{t}^{3} P_{3}^{p h} \xi_{c \rightarrow t}^{T}\right) / g^{2} \\
& \partial \tilde{P}_{2}^{p h} / \partial \kappa_{t}^{3}=\kappa_{v}^{1}\left(P_{1}^{p h}\right)^{2} \xi_{c \rightarrow v}^{R}\left(\kappa_{v}^{1} \xi_{c \rightarrow v}^{R}+\kappa_{t}^{1} \xi_{c \rightarrow t}^{T}-\kappa_{t}^{1} \xi_{c \rightarrow v}^{R}\right) / g^{2}
\end{aligned}
$$




$$
g=\kappa_{v}^{1} P_{1}^{p h} \xi_{c \rightarrow v}^{R}+\kappa_{t}^{1} P_{1}^{p h} \xi_{c \rightarrow t}^{T}+\kappa_{t}^{3} P_{3}^{p h} \xi_{c \rightarrow v}^{R}
$$

Accordingly, in the equilibrium state, increasing $\xi_{c \rightarrow v}^{R}$ (decreasing $\xi_{c \rightarrow t}^{T}$ ) and $\kappa_{v}^{1}$ monotonically improves the intensity of luminescence. Also when $\xi_{c \rightarrow v}^{R}>\xi_{c \rightarrow t}^{T}$ increasing $\kappa_{t}^{1}$ and when $\kappa_{v}^{1}>\kappa_{t}^{1}$ increasing $\kappa_{t}^{3}$ monotonically increases the intensity of luminescence.

One can also combine these two optimizations to build a multi-objective problem to find a way for synthesis of luminescent material with faster and more intense response.

Other practical constraints can be added to build up a more realistic problem or for the reasons that will be further clarified in the next section we may try to increase the curvature of the contours in the equilibrium state plane. In general, we are not essentially interested in finding the global optimal solution; however, while we are synthesizing the phosphor, we want to have some information on the effect of each of these parameters on the overall response of the ETM.

\section{Linear coupling of the light sources}

An interesting case in the equilibrium emission occurs if one couples the two light sources. For example, suppose that the two light sources are linearly coupled so that one source is master and the other is slave,

$$
I_{3}=\eta I_{1}+\mu
$$

In this equation $\eta$ and $\mu$ are real constants. Schematic of a typical optical setup, 
employing such coupling, is displayed in Fig. 6. The linear coupling (LC) of the two light sources means that in the equilibrium state plane, possible values for the intensities $I_{1}$ and $I_{2}$ are located along a line. For instance, consider the case depicted in Fig. 7 for which: $\eta \simeq-1.14$ and $\mu \simeq 1010$. If one sweeps the values of $I_{1}$ from $I_{1}^{\text {Min }}=$ $270 \mathrm{nW}$ to $I_{1}^{\operatorname{Max}}=800 \mathrm{nW}$, as it is depicted in Fig. 7(b), the intensity of luminescence would change nonlinearly. This nonlinear behavior is similar to the desired nonlinear curve that is shown in Fig. 1(b). A sufficient condition to get such a nonlinear behavior is having convex contours of constant luminescence in the equilibrium state plane and negative slope of linear coupling $\eta<0$. As we can see in Fig. 7(a) the terminating points of the linear coupling line, points $T_{1}$ and $T_{2}$, lie on the same contour. As a result, the intensity of luminescence is equal for the terminating points in spite of different intensities of illuminations. At point $T_{1}$, the intensity of the charging illumination is high which causes a large population of trapped electrons. Large population of trapped electrons increases the chance of interaction with photons of both wavelengths $\lambda_{1}$ and $\lambda_{3}$. At point $T_{2}$, the intensity of the charging illumination and the population of trapped electrons is low, however, the intensity of the discharging illumination $I_{3}$ is high which increases the chance of interaction with trapped electrons and photons so that the intensities of luminescence on points $T_{1}$ and $T_{2}$ are equal. Because the contours in the equilibrium state plane can only be convex, in the case of linear coupled sources, the intensity of illumination can only be convex. Nevertheless, by changing the parameters of the linear coupling $\eta$ and $\mu$ and the dynamic ranges of 
the intensities, a variety of different convex nonlinear curves are achievable.

\section{Conclusion}

In this paper, we mathematically investigated the dynamics of electron trapping materials and in particular the equilibrium state luminescence of the material under constant simultaneous illuminations. The derived analytical model complements the experimental observations previously reported $[3,4]$. It is shown in this paper that by linear coupling of the two illuminations a variety of different convex nonlinear curves are producible. The nonlinear curves that are developed in this paper can also be produced by other approaches. As an example, one can generate similar curves by using liquid crystals and controlling the polarization of illumination. However, the dynamics of ETM has at least two advantages over a liquid crystal system. First of all, the dynamics that we discussed here is all-electronic in contrast to liquid crystals where the intensity of output changes as a function of physical orientation of molecules. Rotation of molecules limits the speed of such a system. Another advantage is that with available technology one can precisely control and measure exposure. For instance, one can use the Texas instrument digital micro-mirror device (DMD) [11] to digitally control the exposure with precision as high as 10 bits for more than a million independent pixels. Also, high resolution fast CCD and CMOS digital cameras are commercially available that can precisely measure exposure. In contrast, precise control of polarization is still a challenging problem in the design of liquid crystal 
setups.

The energy band diagram of ETMs that we studied in this paper has three quantum levels and the nonlinear curves that are developed are only convex. By generalizing the idea that is presented here and taking advantage of materials with more complicated energy band diagrams (e.g. adding another trap level in the host bandgap), higher order nonlinear curves would be achievable. Considering the high resolution capabilities of ETMs ( > $100 \mathrm{lp} / \mathrm{mm})$ large arrays of nonlinear processing elements could be accommodated in a thin film of this material and exploited in parallel optical signal processing.

\section{Acknowledgments}

This research was supported in part by Army Research Office (ARO) MURI Grant prime DAAD 19-01-0603 via Georgia Institute of Technology subcontract E-18-67764 and in part by Office of Naval Research Grant No. N00014-94-1-0931 and army research office DURIP instrumentation grant No. W911NF-04-1-0177. 
1. N. H. Farhat, Demetri Psaltis. "Optical implementation of associative memory based on models of neural networks," Optical signal processing, Academic Press Inc, 129-162 (1987).

2. S. H. Strogatz, Nonlinear Dynamics and Chaos: With Applications to Physics, Biology, Chemistry and Engineering, ISBN 0-7382-0453-6.

3. R. Pashaie, N. H. Farhat. "Dynamics of electron-trapping materials under blue light and near-infrared exposure: a new model," J. Opt. Soc. Am. B, 24, 19271941 (2007).

4. R. Pashaie, N. H. Farhat, "Realization of Receptive Fields with Excitatory and Inhibitory Responses on the Equilibrium-State Luminescence of Electron Trapping Material Thin Film", Opt. lett., 32, 1501-1503 (2007).

5. Z. Hua, L. Salamanca-Riba, M. Wuttig, and P. K. Soltani. "Temperature dependence of photoluminescence in $S r S: E u^{2+}, S m^{3+}$ thin films," J. Opt. Soc. Am. B 10, 1464-1469 (1993).

6. S. Jutamulia, G. Stori, J. Lindmayer, W. Seiderman. "Use of electron trapping materials in optical signal processing. 1: parallel Boolean logic," Appl. Opt. 29, 4806-4811 (1990).

7. S. Jutamulia, G. Storti, J. Lindmayer, and W Seiderman. "Optical Information Processing Systems and Architectures," B. Javidi, ed., Proc. Soc. Photo-Opt. 
Instrum. Eng. 1151, 83 (1990).

8. A. D. McAulay, J. Wang, and C. T. Ma," Optical orthogonal neural network associative memory with luminescence rebroadcasting devices," in Proceedings of the IEEE International Conference on Neural Networks, Institute of Electrical and Electronics Engineers, New York, 2, 483-485 (1989).

9. J. Lindmayer. "A new erasable optical memory," Solid State Technol. 31, 135-138 (1988).

10. Z. Wen, N. Farhat. "Dynamics of electron trapping materials for use in optoelectronic neurocomputing," Appl. Opt., 32, 7251-7265 (1993).

11. D. Dudley, W.M. Duncan, J. Slaughter. "Emerging Digital Micromirror Device (DMD) Applications," Proc. SPIE, 4985, 14-25 (2003). 
Fig. 1. (a) Optical setup. A light source illuminates the optical device and a detector measures the intensity of the light that passes through the optical device (b) the normally available and the desired curve.

Fig. 2. Energy bands and optical mechanism of electron trapping materials. Interaction of photons with wavelength $\lambda_{1}$ can excite electrons from valance band to conduction band. Some of these electrons return to the valance band and release their extra energy in the form of spontaneous emission at wavelength $\lambda_{2}$ while others tunnel to the trap energy level. Interaction of photons with wavelengths $\lambda_{1}$ and $\lambda_{3}$ with trapped electrons can detrap some of the trapped electrons.

Fig. 3. System of water tanks as a similar dynamics in fluid mechanics to the optical mechanism of ETMs. In this system the depth of water, $h_{1}(t)$, in tank-1 and, $h_{2}(t)$, tank-2 are dual of the density of the electrons in the trap level and the valance band, respectively. If the cross-sectional areas of the tanks are equal and $F(t)$ represents the inflow of water from the faucet, $D^{1}(t)$ and $D^{2}(t)$ are the outflow of water from drain-1 and drain-2, and $P(t)$ is a function representing the pump action, then the state equations of this system are: $d h_{1}(t) / d t=a F(t) h_{2}(t)-b D^{1}(t) h_{1}(t)-c D^{2}(t) h_{1}(t)$, $d h_{2}(t) / d t=e D^{1}(t) h_{1}(t)+f D^{2}(t) h_{1}(t)-h P(t) h_{2}(t)$, and $h_{1}(t)+h_{2}(t)=$ constant. where the parameters a,b,c,e,f,h are positive real numbers. This set of equations is similar to the set of equations that defines the dynamics of ETM (equations (3) and 
$(4))$.

Fig. 4. (a) Typical charging curves of a completely erased ETM under exposure to light with wavelength $\lambda_{1}$. (b) Typical discharging curves of a precharged ETM under exposure to light with wavelength $\lambda_{3}$, and $P_{t}^{e}(t=0)=10^{8}$. In both graphs $\xi_{c \rightarrow v}^{R}=0.1$, $\kappa_{v}^{1}=0.03, \kappa_{t}^{1}=0.3$. In the charging curves $\kappa_{t}^{3}=0.3$ and in the discharging curves $\kappa_{t}^{3}=0.08$. Wavelengths are: $\lambda_{1}=450 \mathrm{~nm}, \lambda_{2}=650 \mathrm{~nm}$, and $\lambda_{3}=1300 \mathrm{~nm}$.

Fig. 5. (a) Three dimensional plot of the monotonically increasing concave function $f\left(P_{1}^{p h}, P_{3}^{p h}\right)$. (b)The equilibrium state plane of ETM where contours of constant luminescence are plotted as function of the intensities of the two illuminations. In both graphs the material parameters are: $\kappa_{v}^{1}=0.03, \alpha=0.1$, and $\beta=1.0$. Wavelengths are: $\lambda_{1}=450 \mathrm{~nm}, \lambda_{2}=650 \mathrm{~nm}$, and $\lambda_{3}=1300 \mathrm{~nm}$.

Fig. 6. (a) Schematic of a typical optical setup. A thin film of ETM is exposed to two light sources with wavelengths $\lambda_{1}$ and $\lambda_{3}$. In this setup, the first light source is the master and the second one is the slave which is linearly coupled to the first one. Acronyms O.F., LC, and D stand for optical filter, linear coupling system, and photodetector, respectively.

Fig. 7. Linear coupling of two sources and the corresponding nonlinear curve. 


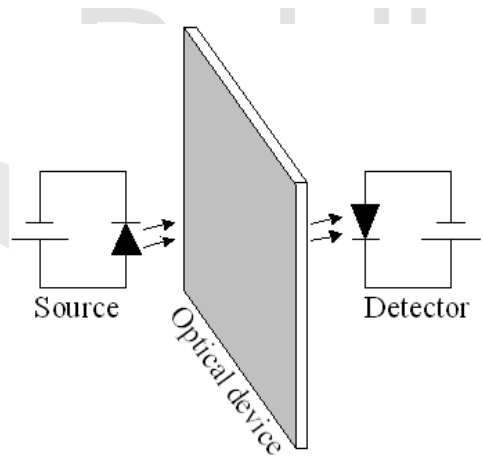

(a)

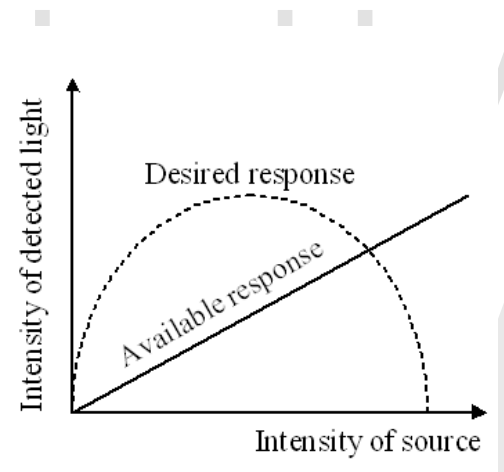

(b)

Fig. 1. (a) Optical setup. A light source illuminates the optical device and a detector measures the intensity of the light that passes through the optical device (b) the normally available and the desired curve. 


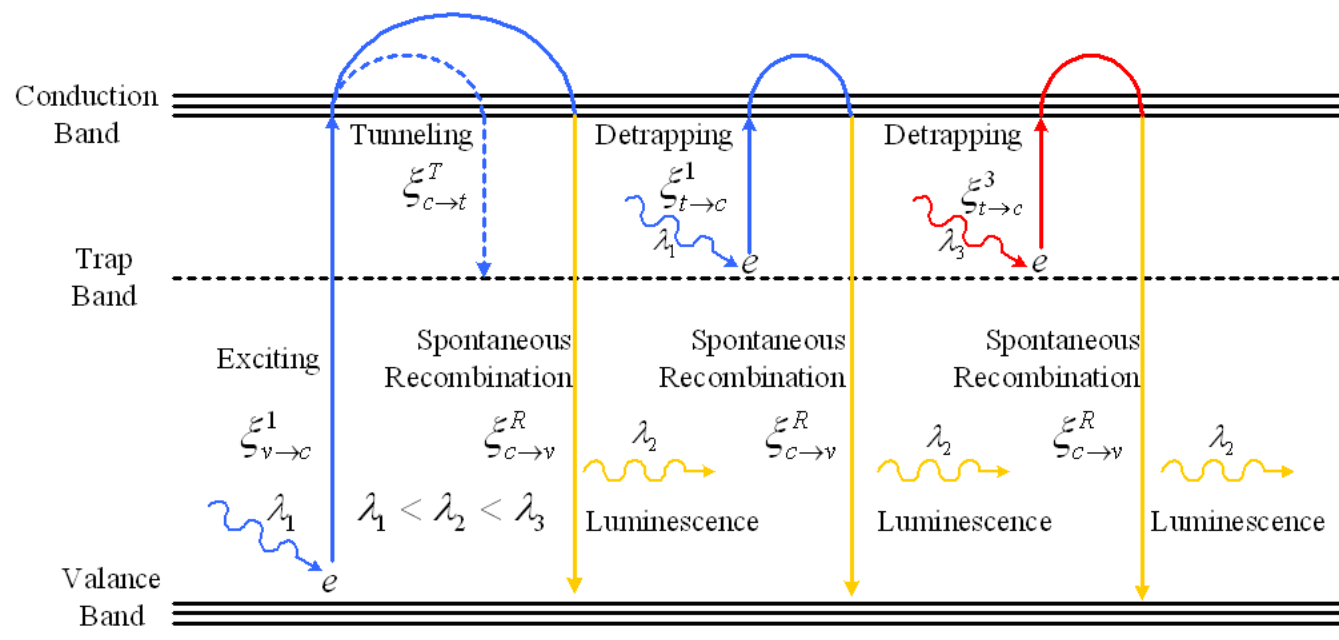

Fig. 2. Energy bands and optical mechanism of electron trapping materials.

Interaction of photons with wavelength $\lambda_{1}$ can excite electrons from valance band to conduction band. Some of these electrons return to the valance band and release their extra energy in the form of spontaneous emission at wavelength $\lambda_{2}$ while others tunnel to the trap energy level. Interaction of photons with wavelengths $\lambda_{1}$ and $\lambda_{3}$ with trapped electrons can detrap some of the trapped electrons. 


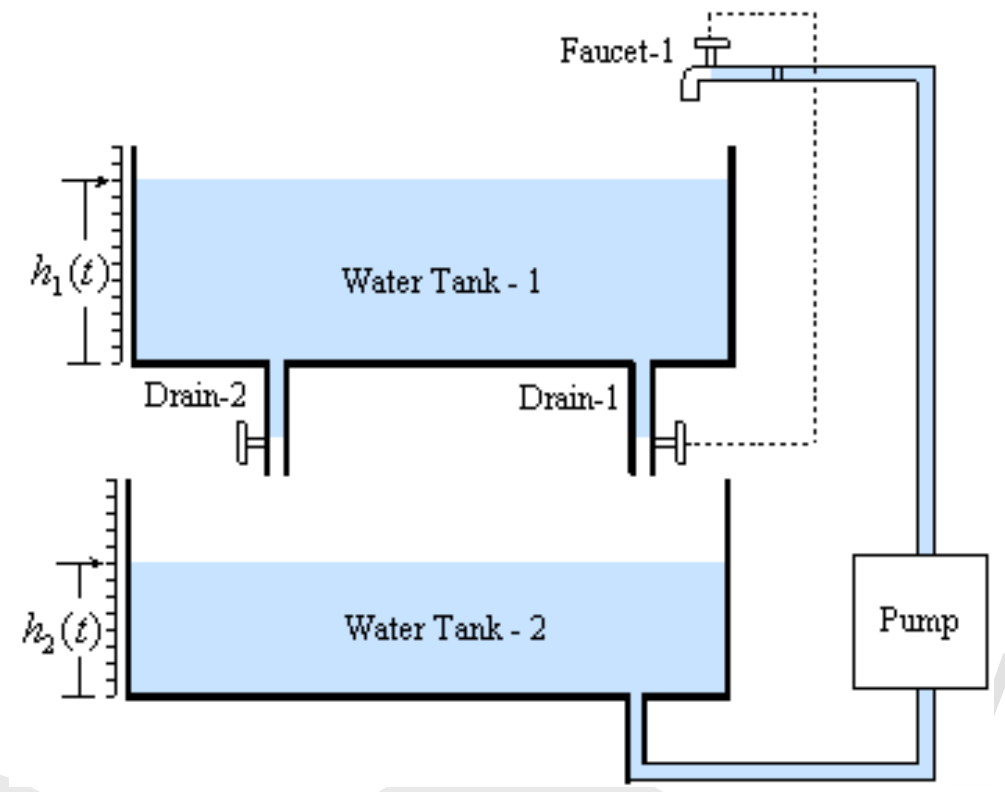

Fig. 3. System of water tanks as a similar dynamics in fluid mechanics to the optical mechanism of ETMs. In this system the depth of water, $h_{1}(t)$, in tank-1 and, $h_{2}(t)$, tank-2 are dual of the density of the electrons in the trap level and the valance band, respectively. If the cross-sectional areas of the tanks are equal and $F(t)$ represents the inflow of water from the faucet, $D^{1}(t)$ and $D^{2}(t)$ are the outflow of water from drain-1 and drain-2, and $P(t)$ is a function representing the pump action, then the state equations of this system are: $d h_{1}(t) / d t=a F(t) h_{2}(t)-b D^{1}(t) h_{1}(t)-c D^{2}(t) h_{1}(t), d h_{2}(t) / d t=$ $e D^{1}(t) h_{1}(t)+f D^{2}(t) h_{1}(t)-h P(t) h_{2}(t)$, and $h_{1}(t)+h_{2}(t)=$ constant. where the parameters a,b,c,e,f,h are positive real numbers. This set of equations is similar to the set of equations that defines the dynamics of ETM (equations (3) and (4)). 

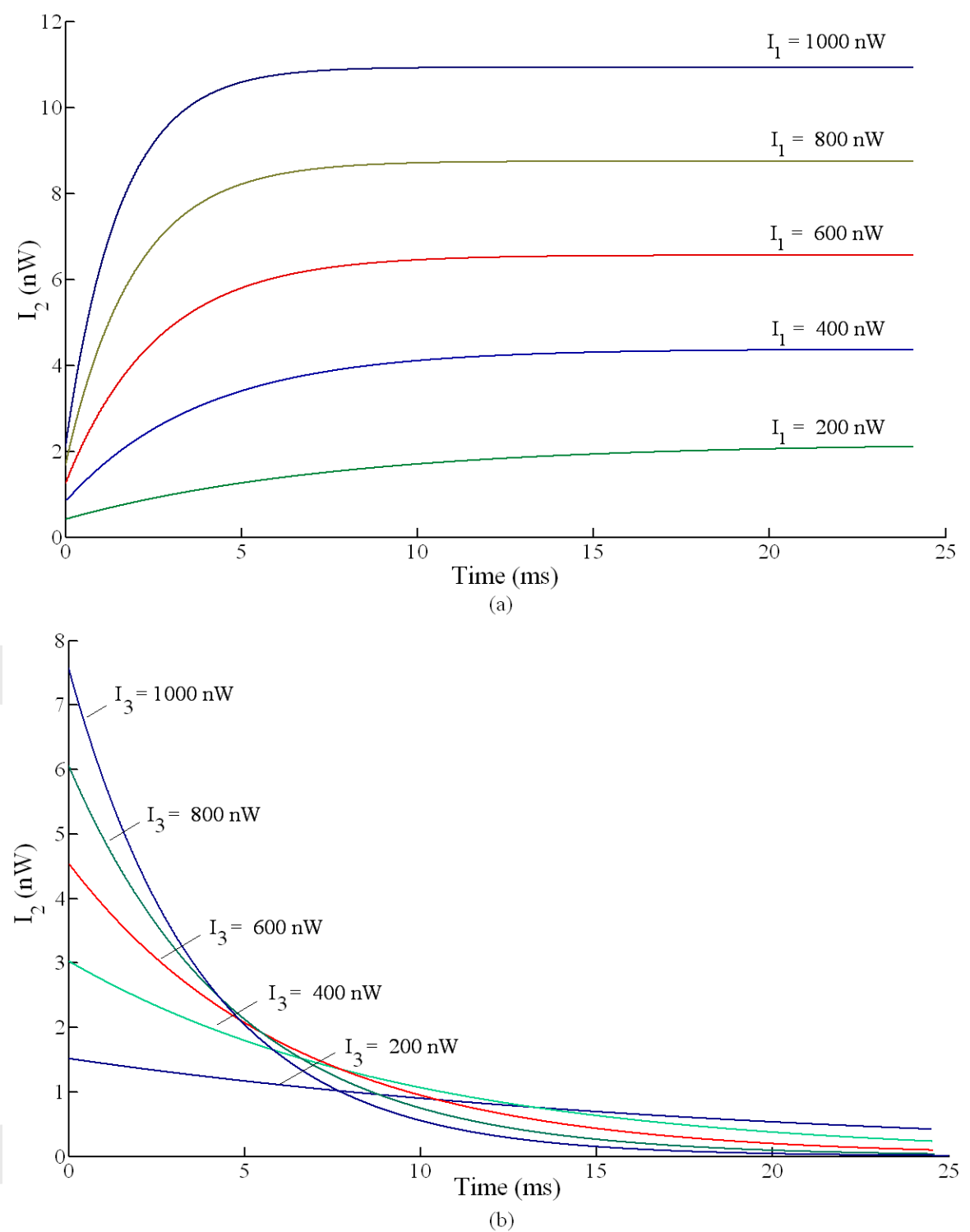

Fig. 4. (a) Typical charging curves of a completely erased ETM under exposure to light with wavelength $\lambda_{1}$. (b) Typical discharging curves of a precharged ETM under exposure to light with wavelength $\lambda_{3}$, and $P_{t}^{e}(t=0)=10^{8}$. In both graphs $\xi_{c \rightarrow v}^{R}=0.1, \kappa_{v}^{1}=0.03, \kappa_{t}^{1}=0.3$. In the charging curves $\kappa_{t}^{3}=0.3$ and in the discharging curves $\kappa_{t}^{3}=0.08$. Wavelengths are: $\lambda_{1}=450 \mathrm{~nm}, \lambda_{2}=650 \mathrm{~nm}$, and $\lambda_{3}=1300 \mathrm{~nm}$. 


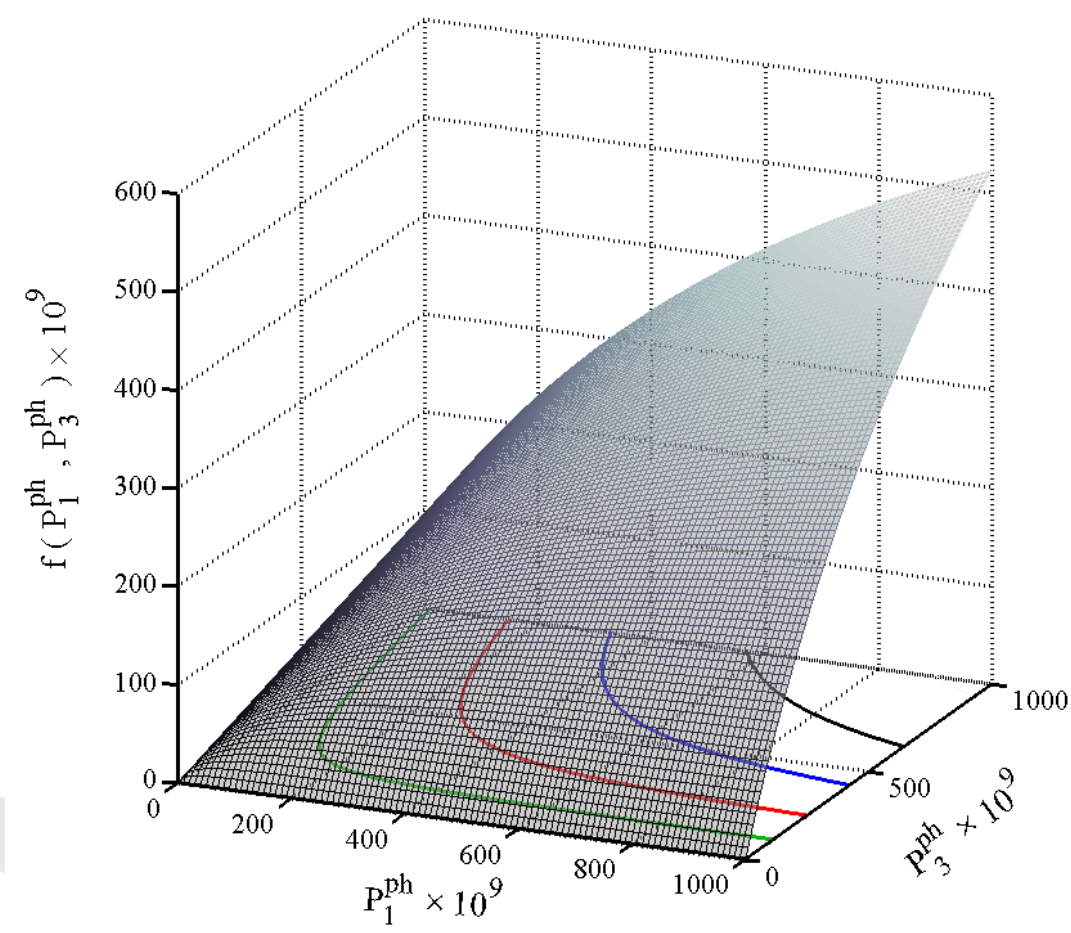

(a)

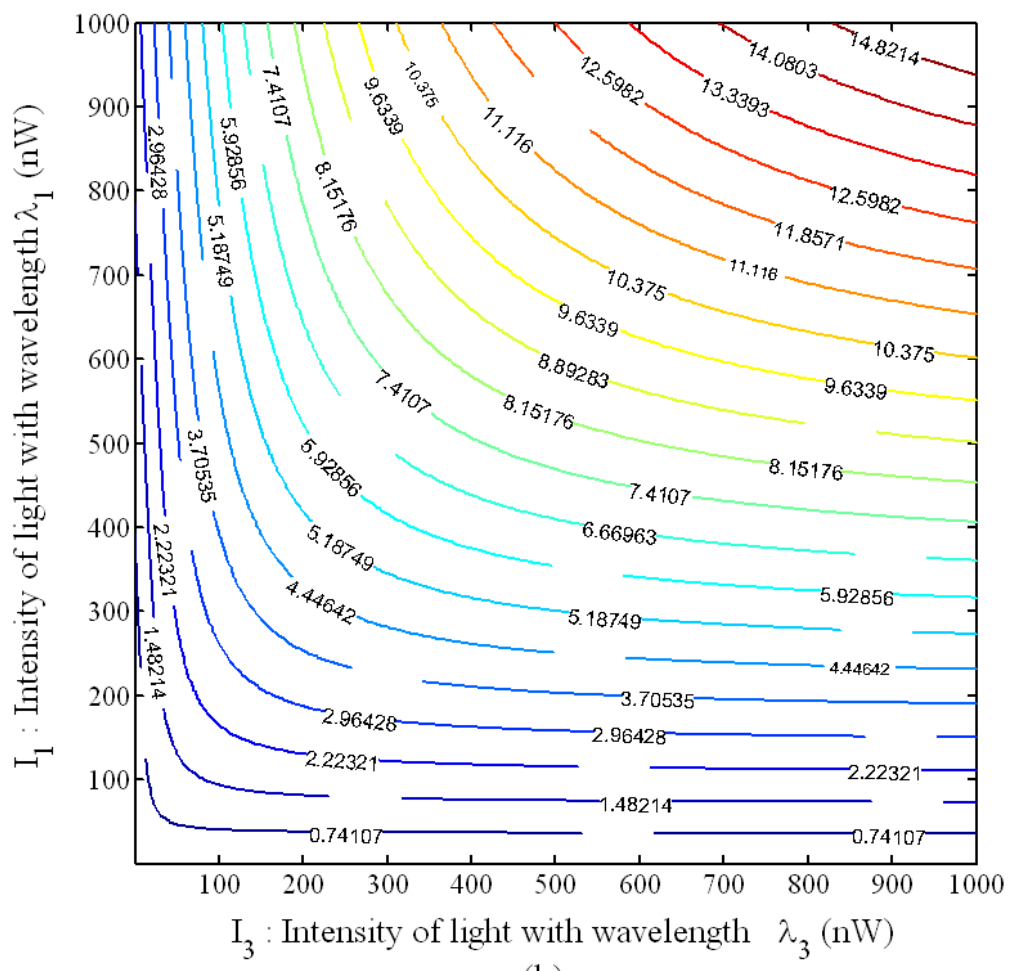

(b)

Fig. 5. (a) Three dimensional plot of the monotonically increasing concave function $f\left(P_{1}^{p h}, P_{3}^{p h}\right)$. (b)The equilibrium state plane of ETM where contours of constant luminescence are plotted as function of the intensities of the two 


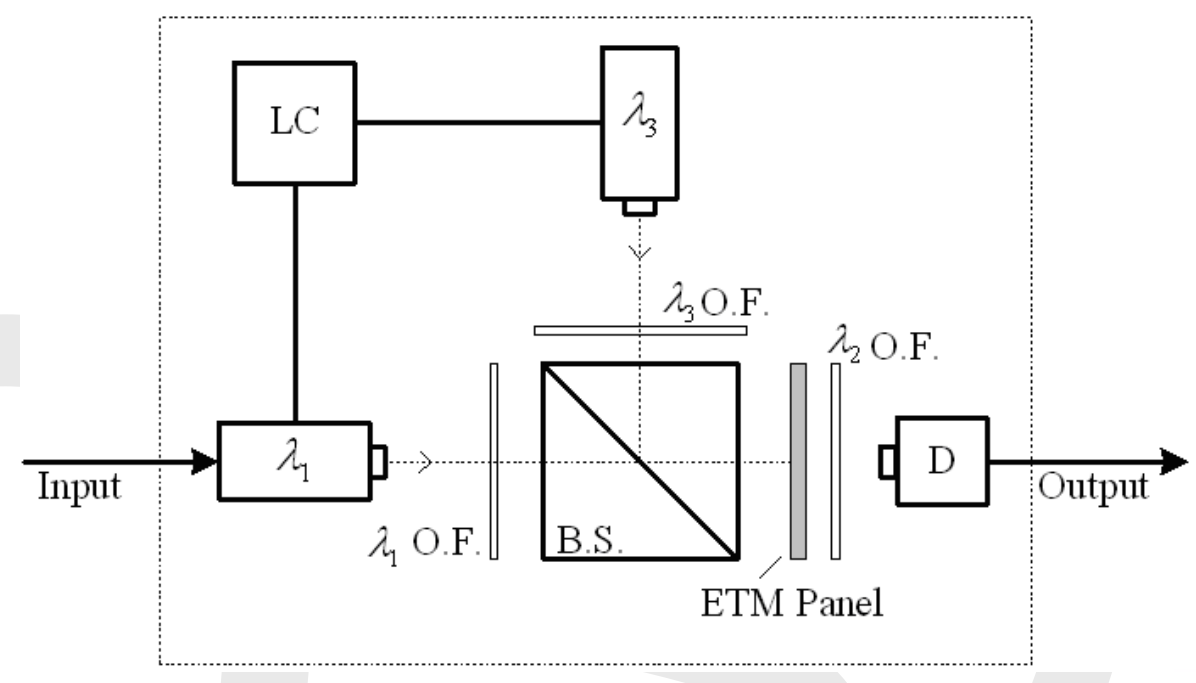

Fig. 6. (a) Schematic of a typical optical setup. A thin film of ETM is exposed to two light sources with wavelengths $\lambda_{1}$ and $\lambda_{3}$. In this setup, the first light source is the master and the second one is the slave which is linearly coupled to the first one. Acronyms O.F., LC, and D stand for optical filter, linear coupling system, and photodetector, respectively. 


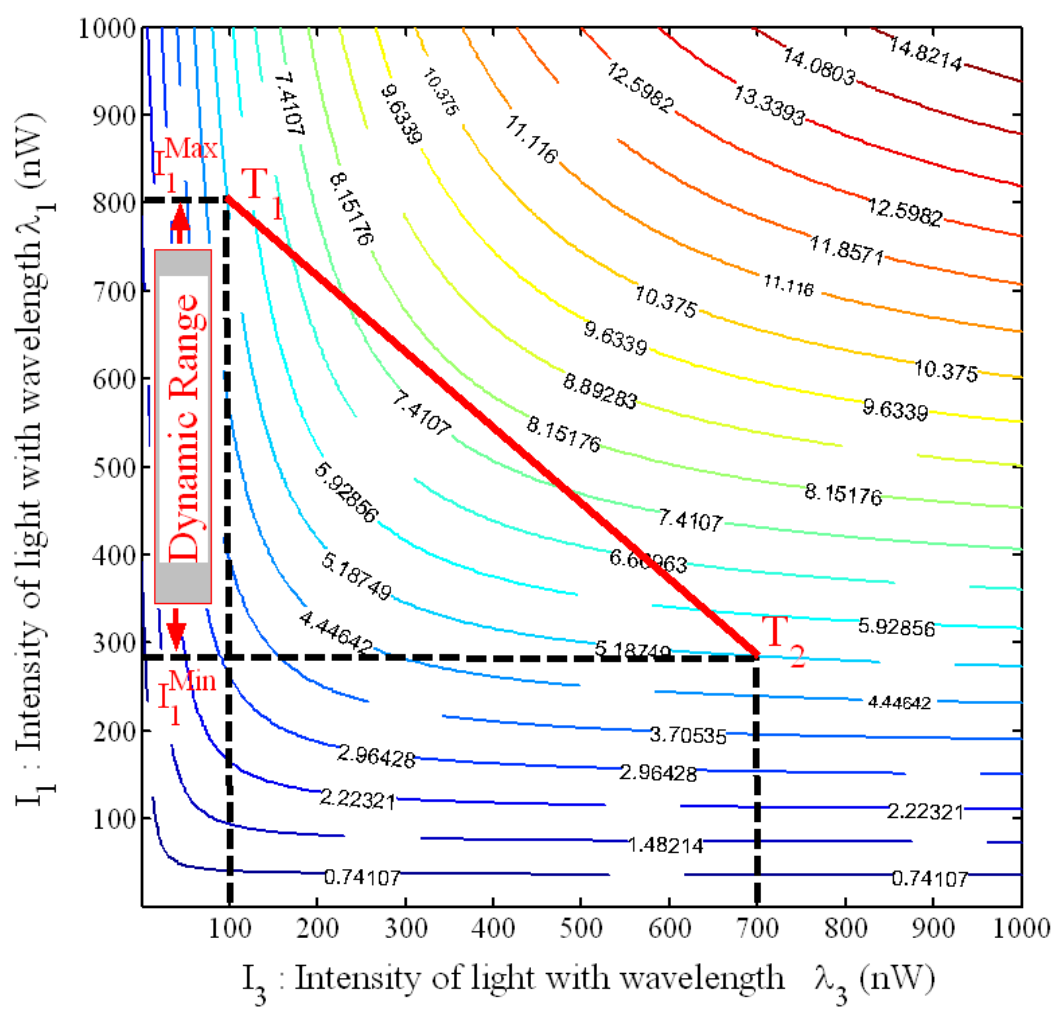

(a)

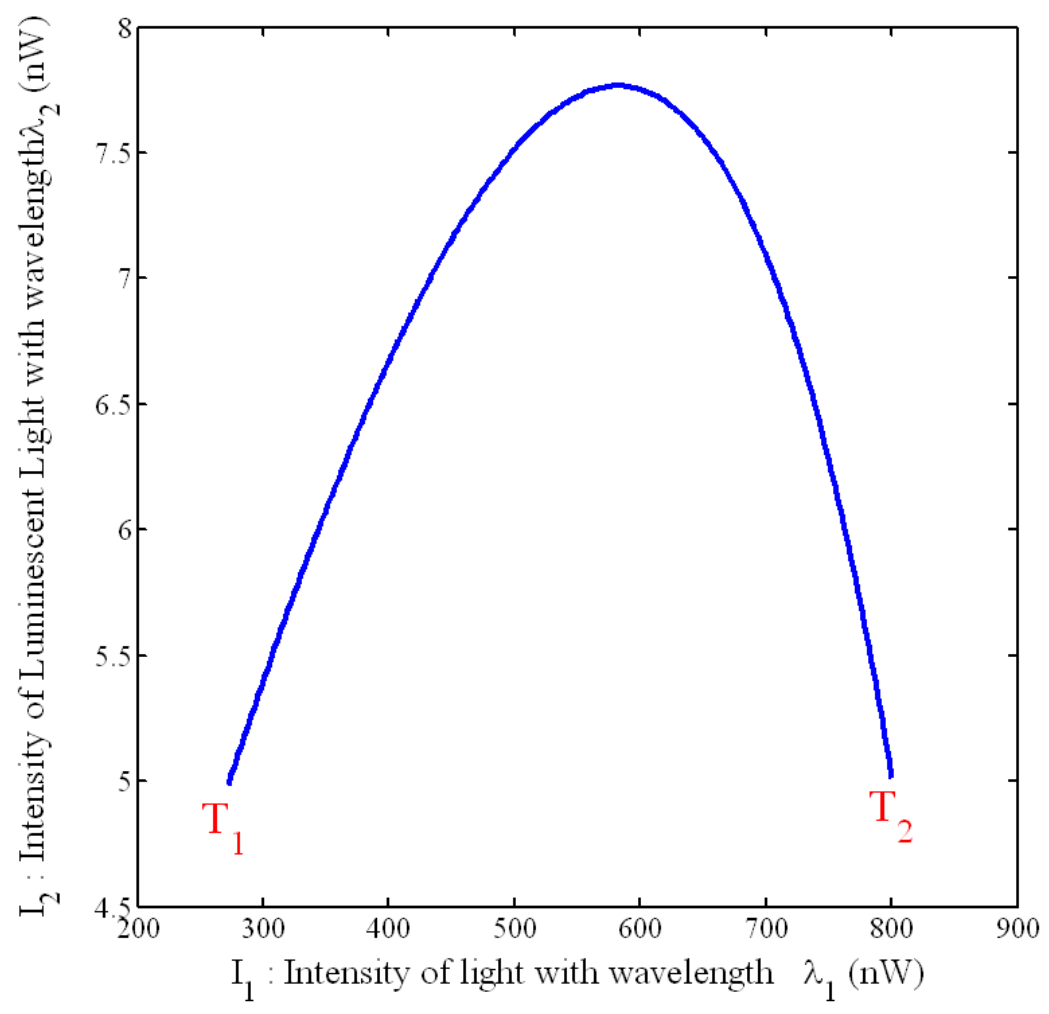

(b)

Fig. 7. Linear coupling of two sources and the corresponding nonlinear curve. 